\title{
Circular dichroism in high harmonic generation from chiral molecules
}

\author{
Yoichi Harada, Eisuke Haraguchi, Keisuke Kaneshima, and Taro Sekikawa* \\ Department of Applied Physics, Hokkaido University, Kita 13 Nishi 8, Kita-ku, Sapporo 060-8628, \\ Japan
}

\begin{abstract}
Circularly polarized high harmonic generation from a chiral molecule was found to significantly depend both on the chirality and on the rotating direction of the circularly polarized counter-rotating two-color driving laser fields.
\end{abstract}

\section{Introduction}

Chiral molecules are not superimposable on their mirror images, called enantiomers. They do not have a symmetry plane nor a center of inversion symmetry. Although the physical properties of the enantiomers are same, the chemical reactivity of chiral molecules exhibits strong enantiomeric selectivity, playing decisive roles in biological systems. Therefore, discerning the enantiomers is important to understand the function of the chirality in biological systems.

The optical approaches such as the circular dichroism of photoabsorption and the optical rotation of the light polarization have been used to distinguish the enantiomers. These effects are induced by the term including the magnetic dipole and, therefore, their magnitude is very small $\left(\sim 10^{-4}\right)$. The molecular chirality has also been detected by the anisotropy of photoelectron emission, which is one or two orders of magnitude larger than those of the conventional techniques.

In this contribution, we report the efficiency of the circularly polarized high harmonic generation (CP-HHG) by two-color bicircular laser field $[1,2]$ depends on the molecular chirality and, therefore, CP-HHG could be a new approach to distinguish the enantiomers. CP-HHG from the single-color single laser beam is prohibited by the conservation of the spin angular momentum. From the viewpoint of the three-step model of HHG, the released electron cannot return to the parent ion under the CP light field, resulting in no emission. In contrast, two-color bicircular laser fields with counter-rotation generate the completely CP high harmonic pulses. The synthesized electric laser field by the fundamental $(\omega)$ and its second harmonic light $(2 \omega)$ has threefold spatio-temporal symmetry and, therefore, the $3 \mathrm{~m}$ th order harmonics are missing from the isotropic media, where $\mathrm{m}$ is a positive integer. The circular polarizations of the $(3 \mathrm{~m}+1)$-th and $(3 \mathrm{~m}+2)$-th harmonics are same as those of $\omega$ and $2 \omega$, respectively, due to the spin angular momentum conservation. For rare gas atoms, the intensity ratio of these neighboring orders was found to depend on the orbital angular

\footnotetext{
${ }^{*}$ Corresponding author: sekikawa@eng.hokudai.ac.jp
} 
momentum symmetry of outmost atomic orbitals correlated with the magnetic dipole induced by the tunnelling of the electron by the laser field $[3,4]$. This stimulated us to observe CP-HHG from chiral molecules. Because they have a fixed magnetic dipole peculiar to the chirality of the molecules, the intensity ratio in the CP-HHP spectrum might depend on the direction of the circular polarization.

\section{Experiment}

We observed the CP-HHG spectra from the enantiomers of limonene [R-limonene and S-limonene]. These enantiomers have different smells. Usually, these are distinguished by optical rotation, of which direction is indicated by the prefixes $\mathrm{R}$ and $\mathrm{S}$. The experimental setup for CP-HHG shown in Fig. 1 is as follows: 1.4-mJ, 35-fs ultrashort laser pulses with a center wavelength of $800 \mathrm{~nm}$ were delivered from a Ti:sapphire laser system at a repletion rate of $1 \mathrm{kHz}$. The two-color counterrotating CP laser field was synthesized by the inline setup called MAZEL-TOV [5]: The linearly polarized $800-\mathrm{nm}$ pulses were partially converted to the $400-\mathrm{nm}$ pulses by a $\beta$-BaB2O4 (BBO) crystal with a vertical polarization to $\omega$. The pulse energies of $\omega$ and $2 \omega$ were 0.9 and $0.5 \mathrm{~mJ}$, respectively. After the compensation of the group delay between $\omega$ and $2 \omega$ by two calcite plates, the polarizations of $\omega$ and $2 \omega$ were changed to circular polarization by a super-achromatic quarter waveplate (SAQW). Here, the circular polarizations of $\omega$ and $2 \omega$ were orthogonal each other and the rotating direction of the laser field was switched by rotating the crystal axis of the SAQW to the incident laser field by 90 degrees.

The synthesized laser field was focused into the gas jet from a glass capillary with an inner diameter of $300 \mu \mathrm{m}$ by a concave mirror with a focal length of $0.5 \mathrm{~m}$. The capillary was set at focus. Otherwise no high harmonics were observed. The enantiomers of limonene molecules were stored in a warmed glass babbler. Limonene vaper was sent with 1.6-atm helium gas through a warmed pipeline to a glass capillary continuously. The spectra of high harmonics were recorded by a magnetic bottle photoelectron spectrometer after converting the high harmonic photons into photoelectrons from krypton atoms. Krypton atoms were employed as target gas because of larger ionization cross sections in spite of the presence of two peaks for each harmonic order owing to the spin-orbit splitting. The photoelectron spectrometer was used in this work just because of the availability of the experimental apparatus.

\section{Results and discussion}

Figures $2 \mathrm{a}$ and $2 \mathrm{~b}$ show the spectra of CP-HHG from R-(+)-limonene and S-(-)limonene, respectively. In this paper, we follow the conventional definition of the leftcircularly polarization as the counterclockwise rotation for an observer facing an oncoming wave. The orange (green) solid lines in Fig. 2 are the CP-HHG spectra generated by the combination of the left (right)-handed $\omega$ and the right(left)-handed $2 \omega$. The red (blue) circular arrows in Fig. 2 indicate the rotating direction of the polarization of $\omega(2 \omega)$. In the spectra, the intensity ratios among the neighboring harmonics were found to depend on the circular polarization. Using the right-handed $\omega$ and the left-handed $2 \omega,(3 \mathrm{~m}+1)$-th harmonics from R-limonene were found to be relatively stronger as shown by the green solid line in Fig. 2a. On the other hand, when the polarization of the driving laser fields was reversed, the same polarization generated a high harmonic spectrum with smaller contrasts in the intensity ratios shown by the orange line. From S-limonene shown in Fig. 2b, the relative intensity ratios among the harmonic orders became totally opposite to the cases of R-limonene. Therefore, these spectral changes both by the direction of circular polarization 
and by the molecular chirality indicate the yields of CP-HHG depend on the chirality of the interacting material with the laser field. The high sensitivity of CP-HHG to chirality might be used for tracing the temporal evolution of chiral molecules in biological activities.

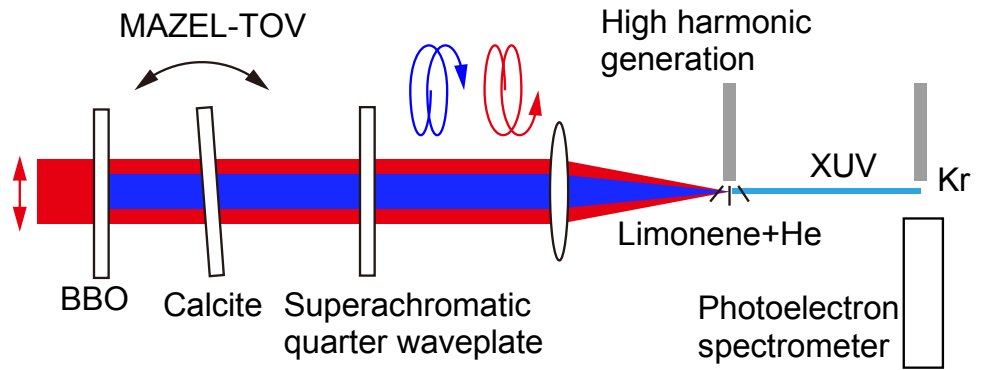

Fig. 1. Experimental setup for the observation of the spectra of CP-HHG. MAZEL-TOV is the optical scheme to generate two-color counterrorating circularly polarized laser field.
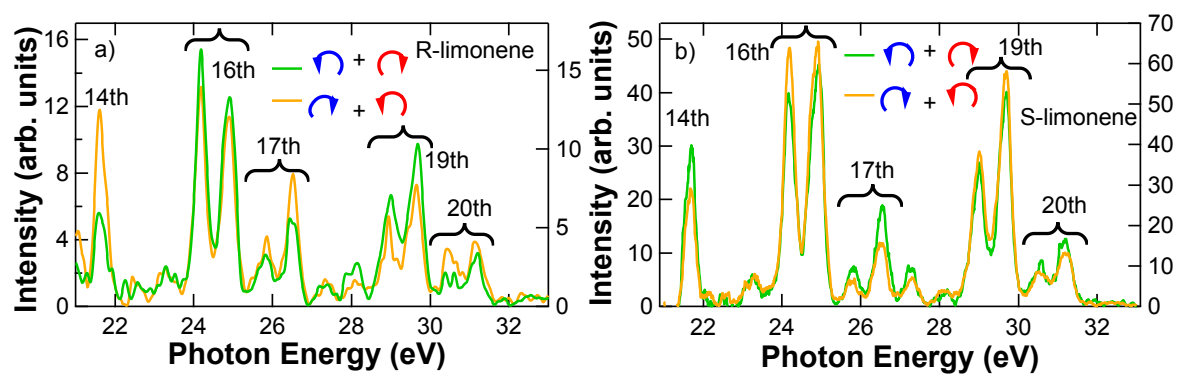

Fig. 2. Spectra of circularly polarized high harmonics generated from a) R-limonene and b) Slimonene. The red and blue arrows indicate the direction of the polarization of the fundamental and the second harmonic used as the driving laser field.

\section{References}

[1] A. Fleischer, O. Kfir, T. Diskin, P. Sidorenko, and O. Cohen, Nature Photon. 8, 543 (2014).

[2] O. Kfir, P. Grychtol, E. Turgut, R. Knut, D. Zusin, D. Popmintchev, T. Popmintchev, H. Nembach, J. M. Shaw, A. Fleischer, H. Kapteyn, M. Murnane, and O. Cohen, Nature Photon. 9, 99 (2015).

[3] L. Medišauskas, J. Wragg, H. v. d. Hart, and M. Y. Ivanov, Phys. Rev. Lett. 115, 153001 (2015).

[4] D. Baykusheva, M. S. Ahsan, N. Lin, and H. J. Wörner, Phys. Rev. Lett. 116, 123001 (2016).

[5] O. Kfir, E. Bordo, G. I. Haham, O. Lahav, A. Fleischer, and O. Cohen, Appl. Phys. Lett. 108, 211106 (2016). 